\title{
Robust Distributed Coordination of Parallel Restored Subsystems in Wind Power Penetrated Transmission System
}

Zhao, Jin; Wang, Hongtao ; Hou, Yunhe; Wu, Qiuwei; Hatziargyriou, N.D.; Chang, Wen; Liu, Yutian

Published in:

IEEE Transactions on Power Systems

Link to article, DOI:

10.1109/TPWRS.2020.2971023

Publication date:

2020

Document Version

Peer reviewed version

Link back to DTU Orbit

Citation (APA):

Zhao, J., Wang, H., Hou, Y., Wu, Q., Hatziargyriou, N. D., Chang, W., \& Liu, Y. (2020). Robust Distributed Coordination of Parallel Restored Subsystems in Wind Power Penetrated Transmission System. IEEE Transactions on Power Systems, 35(4), 3213 - 3223. https://doi.org/10.1109/TPWRS.2020.2971023

\section{General rights}

Copyright and moral rights for the publications made accessible in the public portal are retained by the authors and/or other copyright owners and it is a condition of accessing publications that users recognise and abide by the legal requirements associated with these rights.

- Users may download and print one copy of any publication from the public portal for the purpose of private study or research.

- You may not further distribute the material or use it for any profit-making activity or commercial gain

- You may freely distribute the URL identifying the publication in the public portal 


\title{
Robust Distributed Coordination of Parallel Re- stored Subsystems in Wind Power Penetrated Trans- mission System
}

\author{
Jin Zhao, Student Member, IEEE, Hongtao Wang, Senior Member, IEEE, Yunhe Hou, Senior Mem- \\ ber, IEEE, Qiuwei Wu, Senior Member, IEEE, N. D. Hatziargyriou, Fellow, IEEE, Wen Zhang, Mem- \\ ber IEEE and Yutian Liu, Seninor Member, IEEE
}

\begin{abstract}
Several parallel restored subsystems exist in the last stage of the build-up restoration process of bulk power systems. The whole system restoration is completed when these subsystems are reconnected and their internal loads are recovered. This paper proposes a robust distributed coordination scheme to achieve reconnection and load recovery of parallel restored subsystems in wind power penetrated transmission systems. First, robust distributed restoration models are constructed considering individual subsystems under uncertain conditions. Then, an inner-outer nested upper-lower (IOUL) iterative algorithm is developed to solve the constructed models. The robust distributed coordination can be realized according to convergence. The proposed algorithm solves the complex non-convex model with uncertain variables by iteratively solving the small-scale mixedinteger linear programming (MILP) problem. The robust distributed restoration scheme allows independent decision-making within subsystems and ensures the feasibility of the distributed restoration strategy under uncertain conditions. The effectiveness of the proposed method is validated using three interconnected 6bus systems and the IEEE 118-bus system, showing improved computational efficiency and restoration acceleration.

Index Terms-Bulk power system, distributed optimization, power system restoration, sectionalized restoration, wind power penetration.
\end{abstract}

\section{INTRODUCTION}

$\mathrm{P}$ OWER system restoration is the recovery process after a complete or partial collapse of a power system [1]. Restoring power services quickly after a blackout is crucial for power system resilience [2]. In parallel restoration strategies, the large-scale transmission system restoration process needs to coordinate a number of subsystems during the build-up process. This is essential, since in the early stages of restoration, the blackout area is divided into several sectionalized subsystems to achieve their parallel power system restoration (PPSR)

This work was supported by the Science and Technology Foundation of SGCC (Research on Key Sub-station Control Technology for Rapid Recovery of AC/DC Hybrid Power Grid) (SGSDDK00KJJS1800084) (Corresponding author: Hongtao Wang, Qiuwei $\mathrm{Wu}$ ).

J. Zhao, H. Wang, W. Zhang and Y. Liu are with the Key Laboratory of Power System Intelligent Dispatch and Control of Ministry of Education (Shandong University), Jinan 250000, China. (e-mail: jinzhao_sdu@mail.sdu. edu.cn; whtwhm@sdu.edu.cn; zhangwen@sdu.edu.cn; liuyt@sdu.edu.cn).

Y. Hou is with the Department of Electrical and Electronics, the University of Hong Kong, Hong Kong SAR, China and Shenzhen Institute of Research and Innovation, the University of Hong Kong, Shenzhen, China. (e-mail: yhhou@eee.hku.hk).

Q. Wu is with the Center for Electric Power and Energy, Department of Electrical Engineering, Technical University of Denmark, Kgs. Lyngby, DK 2800 (e-mail: qw@elektro.dtu.dk).

N. D. Hatziargyriou is with the National Technical University of Athens, Athens 15773, Greece (e-mail: nh@power.ece.ntua.gr).
[3]-[5]. Thus, at the end of the PPSR stage, several parallel restored subsystems exist. With high availability of distributed (RESs) resources with black-start capability, such as wind turbines [6] and micro-grids [7], the number of potential individual subsystems also increases. These separate energized subsystems need to be reconnected in the last restoration stage to rebuild the bulk system [1], [3]. Restoration conditions within the various subsystems differ according to their individual generator ramping capability, RES types and penetration and load recovery conditions. Effective coordination of parallel restored subsystems is of vital importance to achieve the bulk system reconnection and accelerate the whole restoration process.

The role of tie-lines is critical for the coordination of the energized subsystems [8], [9]. Ref. [8] identified these roles and proposes a tie-line utilization strategy for different restoration stages. In [9], a centralized method for tie-line utilization is employed based on a central coordination entity with explicit models of subsystems. However, the PPSR process involves several isolated individual grids [1], [3], [10] with restoration plans realized by different independent system operators (ISOs) [11]. Considering the incomplete data exchange among the different ISOs and the data processing burden of a central control center [12], [13], a distributed method is more suitable to coordinate the parallel restored subsystems. Using a distributed scheme, subsystems can make independent restoration decisions, while coordination based on boundary information on tie-lines is possible.

An important factor that should be considered in the restoration of power systems with high penetration of wind power is uncertainty [14]-[16]. Using stochastic programming [14], robust optimization [15] and risk constrained methods [16], uncertainty problem in the transmission restoration process is generally taken into account in a centralized way. To achieve a distributed scheme, the analytical target cascading (ATC) algorithm [17], [18], without requiring convexity of the model [19], is suitable for restoration models with integer variables. In the ATC based method, consistency of shared boundary variables between different subsystems is necessary for maintaining the feasibility of solutions [17], [18]. However, the boundary values obtained by the ATC method [12], [13], [19] may differ due to uncertainties in load and wind power output. Moreover, the step-by-step restoration process aggravates this mismatch [20] leading to not feasible results and security problems. Thus, for the wind power penetrated restoration, the feasibility of the distributed coordination scheme under uncer- 
tainties must be ensured requiring a robust distributed scheme able to guarantee the feasibility of the distributed coordination result under uncertainties.

In this paper, a robust distributed approach is proposed to coordinate parallel restored subsystems for wind power penetrated transmission system restoration. First, a coordinatorsubsystem decision-making structure is built with robust distributed optimization models. Then, the ATC based algorithm is enhanced with an inner-outer nested upper-lower (IOUL) iterative loop to solve the proposed robust distributed models. Finally, the robust distributed restoration strategy is obtained by iteratively solving small-scale mixed-integer linear programming (MILP) models.

The contributions of this paper can be summarized as follows: 1) A robust distributed coordination scheme is proposed to coordinate parallel restored subsystems for the last stage of the build-up restoration process under uncertainties in wind power penetrated power systems. 2) The ATC based distributed method is improved to ensure the feasibility of the result, even in the worst-case uncertainties. 3) An IOUL algorithm is developed to iteratively solve the robust distributed restoration model. Accordingly, the large scale non-convex restoration model with uncertainty variables is transformed into smallscale parallel MILP models.

The rest of the paper is organized as follows: Section II provides compact forms of robust distributed restoration models with the coordinator-subsystem structure. Detailed models are built in Section III. Section IV presents the IOUL algorithm and information interaction process. Section $\mathrm{V}$ provides case study results and discussions, followed by the conclusions.

\section{Framework of PARAllel Restored Subsystem COORDINATION}

This section introduces the framework of the parallel restored subsystem coordination. The structure of the parallel restored transmission system is described and the shared variables of the tie-lines are defined. The coordinator-subsystem structure is designed followed by robust distributed restoration models in a compact form.

\section{A. Coordination of parallel restored subsystems}

According to the parallel sectionalization approach, several individual subsystems exist in the PPSR process. At the last stage of PPSR process, these parallel subsystems have restarted their generators and energized their transmission lines [4], [5], [10]. However, their loads are not completely restored and the subsystems are isolated. As shown in Fig. 1, it is assumed that each subsystem contains traditional generators, wind farms, loads and a meshed network with different generator ramping up and load recovery conditions. The coordination of parallel restored subsystems aims to balance power supply and demand under different regional restoration conditions and rebuild the interconnected transmission system. Since information of each subsystem cannot be completely shared due to commercial or security consideration, a distributed coordination scheme is preferred.

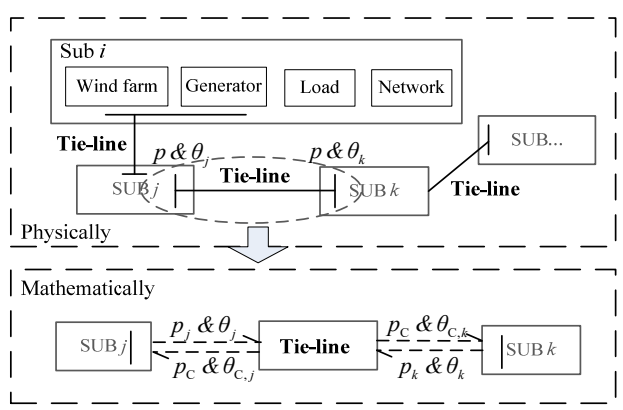

Fig. 1. Boundary shared variables between the tie-line and subsystem.

As shown in Fig.1, the key to realize distributed coordination is the decoupling of shared boundary variables between the tie-line and subsystem. The shared variables to avoid the excessive standing phase angle (SPA) problem [21], are the phase angle $\theta$ at the interconnecting bus and the active power $p$ of the tie-lines. Besides the phase angle, the frequency and voltage differences should also be within acceptable limits. The frequency difference requirement can be satisfied by using the isochronous machine in each subsystem, while local voltage regulation can be used to meet the voltage difference requirement since voltage constraints are included in the optimization model. In Fig. $1, p_{\mathrm{C}}, p_{j}$ and $p_{k}$ are the active power from the tie-line, subsystem $j$ and subsystem $k$, respectively, and $\theta_{j}$ and $\theta_{\mathrm{C}, j}$ represent phase angles at the interconnecting bus of subsystem $j$ and the tie-line connecting subsystem $j$. 'SUB $j$ ' stands for subsystem $j$. For effective parallel subsystem restoration, all the loads should be restored, as soon as possible, to complete the restoration process, i.e. the load recovery at each step of the restoration process should be maximized. [3]. Model (1) represents the load restoration coordination model for the whole system consisting of several parallel subsystems.

$$
\begin{array}{ll}
\operatorname{Max} & \sum_{j \in N_{\mathrm{sub}}} \sum_{i \in N_{\mathrm{L}, j}}\left(c_{\mathrm{L}, j, i} E_{\mathrm{L}, j, i} x_{\mathrm{L}, j, i}\right) \\
\text { s.t. } & \boldsymbol{g}_{j}\left(\boldsymbol{x}_{\mathrm{L}, j}, \boldsymbol{p}_{\mathrm{G}, j}, \boldsymbol{p}_{\mathrm{L}, j}, \boldsymbol{p}_{\mathrm{RE}, j}, \boldsymbol{S}_{\mathrm{B}, j}\right) \leq 0 \\
& \boldsymbol{h}_{j}\left(\boldsymbol{x}_{\mathrm{L}, j}, \boldsymbol{p}_{\mathrm{G}, j}, \boldsymbol{p}_{\mathrm{L}, j}, \boldsymbol{p}_{\mathrm{RE}, j}, \boldsymbol{S}_{\mathrm{B}, j}\right)=0 \\
& \boldsymbol{g}_{C_{C_{i}}}\left(\boldsymbol{S}_{C_{i, j},}, \boldsymbol{S}_{C_{i}, k}\right) \leq 0 \\
& \boldsymbol{h}_{C_{i}}\left(\boldsymbol{S}_{C_{i, j},}, \boldsymbol{S}_{C_{i, k}}\right)=0 \\
& \boldsymbol{\sigma}_{j}=\boldsymbol{S}_{C_{i, j}}-\boldsymbol{S}_{\mathrm{B}, j}=0 \\
& \boldsymbol{\sigma}_{k}=\boldsymbol{S}_{C_{i, k}}-\boldsymbol{S}_{\mathrm{B}, k}=0 \\
& \boldsymbol{\sigma}_{C_{i}}=\left[\sigma_{j}, \boldsymbol{\sigma}_{k}\right] \\
& \forall j, k \in N_{\mathrm{Sub}}, j \neq k, C_{i} \in N_{\mathrm{tie}}
\end{array}
$$

where $x_{\mathrm{L}, j, i}, E_{\mathrm{L}, j, i}$ and $c_{\mathrm{L}, j, i}$ are the binary variable of load pickup decision, expected load amount and the corresponding weighting coefficients of load $i$ at subsystem $j, \boldsymbol{x}_{\mathrm{L}, j}, \boldsymbol{p}_{\mathrm{G}, j}, \boldsymbol{p}_{\mathrm{L}, j}$ and $\boldsymbol{p}_{\mathrm{RE}, j}$ are sets of load pickup decision variables, active power outputs of generators, uncertain load amounts and uncertain active outputs of wind farms. $\boldsymbol{S}_{\mathrm{B}, j}$ is the set of shared variables $\theta$ and $p$ for subsystem $j, \boldsymbol{S}_{C i, j}$ and $\boldsymbol{S}_{C i, k}$ contain shared variables of the tie-line $C_{i}$ connecting subsystems $j$ and $k$, respectively, $\boldsymbol{\sigma}_{j}$ and $\boldsymbol{\sigma}_{C i}$ are sets of shared variable mismatches at one side of the tie-line connecting subsystems $j$ and at tie-line $C_{i} . \boldsymbol{g}_{j}()$ and $\boldsymbol{h}_{j}()$ represent the inequality and equality constraints of generator outputs, AC power flow, boundary variables, voltage and frequency of subsystem $j$, and $\boldsymbol{g}_{C i}()$ and $\boldsymbol{h}_{C i}()$ are boundary variable limits and variable consistency con- 
straints of tie-line $C_{i}$. $N_{\mathrm{Sub}}, N_{\mathrm{L}, j}$ and $N_{\text {tie }}$ are sets of subsystems, loads in subsystem $j$ and tie-lines, respectively. Note that the load amount variables in $\boldsymbol{p}_{\mathrm{L}, j}$ and wind farm output variables in $\boldsymbol{p}_{\mathrm{RE}, j}$ are all uncertain variables integrated in constraints. $E_{\mathrm{L}, j, i}$ with a fixed value is just used in the objective function.

\section{B. Robust distributed coordination structure}

Based on Model (1), the optimization model can be divided according to parallel subsystems if tie-line constraints are separated. Using the Lagrangian decomposition method [17], the constraint set $\boldsymbol{\sigma}=0$ is transformed into $\Phi(\boldsymbol{\sigma})$ and is further added to the objective function. The augmented Lagrangian method with a quadratic penalty term [18] is used to form $\Phi(\boldsymbol{\sigma})$ where $\boldsymbol{v}_{j}$ and $\boldsymbol{w}_{j}$ are sets of penalty multipliers for boundary variables decoupling of subsystem $j$ and are updated during the iterative process. The symbol $\circ$ represents the Hadamard product which is an entry-wise multiplication of two vectors.

$$
\Phi(\boldsymbol{\sigma})=\boldsymbol{v} \boldsymbol{\sigma}^{\mathrm{T}}+\left\|\boldsymbol{w} \circ \boldsymbol{\sigma}^{\mathrm{T}}\right\|_{2}^{2}=\sum_{j \in N_{\text {Sub }}}\left(\boldsymbol{v}_{j} \boldsymbol{\sigma}_{j}+\left\|\boldsymbol{w}_{j} \circ \boldsymbol{\sigma}_{j}\right\|_{2}^{2}\right)
$$

With uncertain variables $\boldsymbol{p}_{\mathrm{L}, j}$ and $\boldsymbol{p}_{\mathrm{RE}, j}$, Model (1) is transformed into robust distributed models (3)-(4). Model (3) is the tie-line optimization model which can be regarded as the coordinator, and Model (4) is the optimization model of parallel subsystems. Model (4) is a typical two-stage robust optimization model. The first stage includes the first $\min$ term of the objective in (4) and load pickup variable related constraints, while the second stage contains the max-min term in the objective and all the rest constraints. The subsystem model (4) maximizes regional load recovery at the first stage and minimizes the mismatch of boundary shared variables in the worst-case under which uncertain wind power output and load amount deteriorate the boundary consistency at the second stage. With a boundary consistency threshold, the feasibility of the distribution method under uncertain conditions can be guaranteed.

$$
\begin{aligned}
& \text { Coordinator: } \operatorname{Min} \sum_{\forall C_{i} \in N_{\text {tie }}} \Phi_{\mathrm{C}}\left(\sigma_{C_{i}}\right) \\
& \text { s.t. } \boldsymbol{g}_{C_{i}}\left(\boldsymbol{S}_{\mathrm{C}_{i}, j}, \boldsymbol{S}_{C_{i}, k}\right) \leq 0 \\
& \boldsymbol{h}_{C_{i}}\left(\boldsymbol{S}_{C_{i}, j}, \boldsymbol{S}_{C_{i}, k}\right)=0 \\
& j, k \in N_{\text {Sub }}, j \neq k, \forall C_{i} \in N_{\text {tie }} \\
& \text { Subsystem : } \operatorname{Min}_{\mathbf{x}_{\mathrm{L}, j}}-\sum_{i \in N_{\mathrm{L}, j}}\left(c_{\mathrm{L}, j, i} E_{\mathrm{L}, j, i} x_{\mathrm{L}, j, i}\right)+\operatorname{Max}_{\boldsymbol{P}_{\mathrm{L}}, \boldsymbol{P}_{\mathrm{W}}} \operatorname{Min}_{\mathrm{B}_{, j}, \boldsymbol{p}_{\mathrm{G}, j}} \Phi_{j}\left(\sigma_{j}\right) \\
& \text { s.t. } \boldsymbol{g}_{j}\left(\boldsymbol{x}_{\mathrm{L}, j}, \boldsymbol{p}_{\mathrm{G}, j}, \boldsymbol{p}_{\mathrm{L}, j}, \boldsymbol{p}_{\mathrm{RE}, j}, \boldsymbol{S}_{\mathrm{B}, j}\right) \leq 0 \\
& \boldsymbol{h}_{j}\left(\boldsymbol{x}_{\mathrm{L}, j}, \boldsymbol{p}_{\mathrm{G}, j}, \boldsymbol{p}_{\mathrm{L}, j}, \boldsymbol{p}_{\mathrm{RE}, j}, \boldsymbol{S}_{\mathrm{B}, j}\right)=0 \\
& j \in N_{\text {Sub }}
\end{aligned}
$$

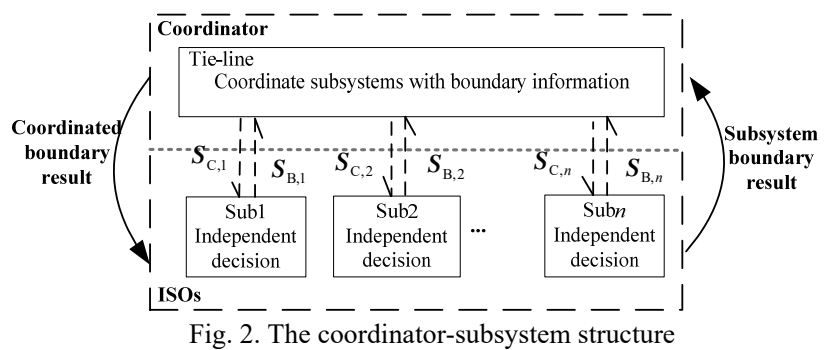

The coordination of parallel subsystems relies on an iterative information interaction process. As shown in Fig. 2, a coordinator-subsystem framework is designed based on the robust distributed models. In this framework, parallel subsys- tems make independent restoration decisions according to Model (4), and submit boundary results to the coordinator. With the boundary information of subsystems, the coordinator performs Model (3) and provides coordinated boundary results to subsystems. The information interaction process continues until the coordinator and subsystems reach boundary consensus. The practical coordinator can be the upper-level dispatch center for different regional ISOs in the bulk system with knowledge of tie-lines.

\section{Modeling OF RoBust Distributed CoORdinAtion}

Considering uncertainties, the overall problem is formulated as distributed robust optimization models in this Section. First, the detailed restoration models of the coordinator and the subsystems are built. Then, the coordinator-subsystem models are transformed into MILP problems.

\section{A. Robust distributed coordinator-subsystem models}

First, the model of the coordinator is formulated.

1) Objective: the objective of the upper-level coordinator is to coordinate all the subsystems by pursuing the consistency of boundary variable values between tie-lines and parallel subsystems. In (5), $p_{C i, j}$ and $\theta_{C i, j}$ are the boundary active power and phase angle of tie-line $C_{i}$ between tie-line $C_{i}$ and subsystem $j, p_{C i, k}$ and $\theta_{C i, k}$ are the ones of tie-line $C_{i}$ between tie-line $C_{i}$ and subsystem $k, p_{\mathrm{B}, j, C_{i}}^{*} p_{\mathrm{B}, k, C_{i},}^{*} \theta_{\mathrm{B}, j, C}^{*}$ and $\theta_{\mathrm{B}, k, C i}^{*}$ are boundary active power and phase angles provided by subsystem $j$ and $k$.

$$
\operatorname{Min} \sum_{C_{i} \in N_{\mathrm{tie}}}\left[\begin{array}{l}
v_{j}\left(p_{C_{i}, j}-p_{\mathrm{B}, j, C_{i}}^{*}\right)+\left\|w_{j} \circ\left(p_{C_{i}, j}-p_{\mathrm{B}, j, C_{i}}^{*}\right)\right\|_{2}^{2} \\
+v_{j}\left(\theta_{C_{i}, j}-\theta_{\mathrm{B}, j, C_{i}}^{*}\right)+\left\|w_{j} \circ\left(\theta_{C_{i}, j}-\theta_{\mathrm{B}, j, C_{i}}^{*}\right)\right\|_{2}^{2} \\
+v_{k}\left(p_{C_{i}, k}-p_{\mathrm{B}, k, C_{i}}^{*}\right)+\left\|w_{k} \circ\left(p_{C_{i}, k}-p_{\mathrm{B}, k, C_{i}}^{*}\right)\right\|_{2}^{2} \\
+v_{k}\left(\theta_{C_{i}, k}-\theta_{\mathrm{B}, k, C_{i}}^{*}\right)+\left\|w_{k} \circ\left(\theta_{C_{i}, k}-\theta_{\mathrm{B}, k, C_{i}}^{*}\right)\right\|_{2}^{2}
\end{array}\right]
$$

2) Constraints of tie-lines. Constraints (6)-(7) comprise active power limits of tie-lines. A maximum phase angle difference is required in (8) to avoid excessive SPA problems between neighboring subsystems.

$$
\begin{gathered}
p_{C_{i}, j}=-p_{C_{i}, k}\left(\forall C_{i} \in N_{\text {tie }}\right) \\
\left|p_{C_{i}, j}\right| \leq p_{C_{i}, \max }\left(\forall C_{i} \in N_{\text {tie }}\right) \\
\left|\theta_{C_{i}, j}-\theta_{C_{i}, k}\right| \leq \theta_{j k, \text { max }} \quad \forall C_{i} \in N_{\text {tie }}
\end{gathered}
$$

Without loss of generality, the load restoration oriented robust distribution model of subsystem $j$ is constructed as follows.

1) Objective: The objective of the subsystem is to restore its own load considering coordination from the coordinator. The first term of (9) is the weighted load restoration amount and the latter term represents coordination with tie-lines. The subsystem model is a two-stage robust optimization with a polyhedral uncertainty set $\Phi$ (10) for uncertain wind farm output and load amount [22]. In (10), $p_{\text {low }, i}$ and $p_{u p, i}$ are lower and upper active powers of the uncertain variable related to node $i$. 


$$
\begin{aligned}
& \operatorname{Min}_{x_{\mathrm{L}, j}}-\sum_{i \in N_{\mathrm{L}, j}}\left(c_{\mathrm{L}, j, i} E_{\mathrm{L}, j, i} x_{\mathrm{L}, j, i}\right) \\
& +\underset{\left(\boldsymbol{P}_{\mathrm{L},}, \boldsymbol{P}_{\mathrm{W}_{j}, j}\right) \in \Phi}{\operatorname{Max}} \operatorname{Min} \sum_{C_{i} \in S u_{j} b_{j}}\left(\begin{array}{l}
v_{j}\left(p_{C_{i}, j}^{*}-p_{\mathrm{B}, j, C_{i}}\right)+v_{j}\left(\theta_{C_{i}, j}^{*}-\theta_{\mathrm{B}, j, C_{i}}\right) \\
+\left\|w_{j} \circ\left(p_{C_{i}, j}^{*}-p_{\mathrm{B}, j, C_{i}}\right)\right\|_{2}^{2}+\left\|w_{j} \circ\left(\theta_{C_{i}, j}^{*}-\theta_{\mathrm{B}, j, C_{i}}\right)\right\|_{2}^{2}
\end{array}\right) \\
& \Phi=\left\{\left(\boldsymbol{P}_{\mathrm{L}, j}, \boldsymbol{P}_{\mathrm{W}, j}\right): p_{\text {low }, i} \leq p_{i} \leq p_{u p, i}, i \in N_{\mathrm{L}, j} \& N_{\mathrm{W}, j}\right\}
\end{aligned}
$$

2) Power balance constraints: The power supply contains the output of traditional generators and wind farms. In the load restoration stage, the conventional generators ramp up with ramp rate $r_{i}$ between upper and lower limits in (11) [23]. Constraint (12) ensures spinning reserve which survives the largest loss of energy contingency. The limit of the restoration step time is shown in (13). Equalities (14) and (15) determine the active and reactive power of each bus in subsystem $j$, respectively. Constraints (16)-(22) are the linearized AC power flow which performs polyhedral relaxation of cosine function and linearization by Taylor series expansion, as detailed in [24].

$$
\begin{gathered}
\max \left\{p_{\mathrm{G}, i, i n i}+r_{i} T_{m}, p_{\mathrm{G}, i, \min }\right\} \leq p_{\mathrm{G}, i} \leq \min \left\{p_{\mathrm{G}, i, i n i}+r_{i} T_{m}, p_{\mathrm{G}, i, \max }\right\} \\
p_{\mathrm{G}, i} \leq \sum_{i^{e} \in N_{\mathrm{G}, j}, i^{*} \neq i}\left(r_{i^{i}} T_{\max }+p_{\mathrm{G}, i^{e}}\right)+\sum_{i \in N_{\mathrm{W}, j}} p_{\mathrm{W}, i}-\sum_{C_{i} \in S u b_{j}} p_{\mathrm{B}, j, C_{i}}-\sum_{i \in N_{\mathrm{L}, j}} x_{\mathrm{L}, i} p_{\mathrm{L}, i} \\
T_{\min } \leq T_{m} \leq T_{\max } \\
p_{n}=-x_{\mathrm{L}, i} p_{\mathrm{L}, i}\left(i \in N_{\mathrm{L}, j}\right)-p_{\mathrm{B}, j, C_{i}}\left(C_{i} \in S u b_{j}\right) \\
+p_{\mathrm{W}, i}\left(i \in N_{\mathrm{W}, j}\right)+p_{\mathrm{G}, i}\left(i \in N_{\mathrm{G}, j}\right)+0 \\
q_{n}=-x_{\mathrm{L}, i} q_{\mathrm{L}, i}\left(i \in N_{\mathrm{L}, j}\right)+q_{\mathrm{G}, i}\left(i \in N_{\mathrm{G}, j}\right)+q_{\mathrm{B}, i}\left(i \in N_{\mathrm{B}, j}\right)+0 \\
p_{n}=\sum_{\langle n, m\rangle \in L_{j}} P_{n m} \forall n \in N_{S u b_{j}} \\
q_{n}=\sum_{\langle n, m\rangle \in L_{j}} Q_{n m} \forall n \in N_{S u b_{j}} \\
P_{n m}=g_{n m}-g_{n m} \cos \theta_{n m}-b_{n m}\left(\theta_{n}-\theta_{m}\right) \\
Q_{n m}=-b_{n m}-g_{n m}\left(\theta_{n}-\theta_{m}\right)+b_{n m} \cos ^{*} \theta_{n m}-b_{n m}\left(\delta_{n}-\delta_{m}\right) \\
\cos ^{*} \theta_{n m} \geq \cos \theta_{\Delta}^{0} \\
\leq-\sin \left(v d-\theta_{\Delta}^{0}\right)\left(\theta_{n}-\theta_{m}-v d+\theta_{\Delta}^{0}\right)+\cos \left(v d-\theta_{\Delta}^{0}\right) \\
v=1,2, \ldots, 2 h \\
V_{n}=1+\delta_{n}(\operatorname{Volts} p . u .)
\end{gathered}
$$

Variables $p_{\mathrm{G}, i}, p_{\mathrm{G}, i, i n i}, p_{\mathrm{G}, i, \min }$ and $p_{\mathrm{G}, i, \max }$ are the active power output, initial active output in the current step, minimum and maximum output in the whole restoration process of generator at node $i$, respectively, $x_{\mathrm{L}, i}$ is the binary variable of the load pickup decision, $p_{\mathrm{L}, i}$ and $p_{\mathrm{W}, i}$ are uncertain load amount and wind farm output at node $i, p_{\mathrm{B}, j, C i}$ is the boundary active power between subsystem $j$ and tie-line $C_{i}, T$ is the load pickup time, $q_{\mathrm{L}, i}, q_{\mathrm{G}, i}$ and $q_{\mathrm{B}, i}$, are reactive power of the load, generator and boundary node $i$, respectively, $p_{n}$ and $q_{n}$ are the active and reactive power injection into bus $n, P_{n m}$ and $Q_{n m}$ are the active and reactive power flow on line $n m, g_{n m}, b_{n m}$ and $\theta_{n m}$ are the conductance, susceptance and phase angle difference of line $n m, \cos ^{*} \theta_{n m}$ is the approximate variable of $\cos \theta_{n m}$, and $V_{n}$ and $\delta_{n}$ are voltage magnitude and voltage magnitude deviation variable of bus $n . N_{\mathrm{G}, j}, N_{\mathrm{L}, j}, N_{\mathrm{W}, j}, N_{\mathrm{B}, j}, N_{\mathrm{Sub} j}$ and $L_{j}$ contain generator nodes, load nodes, wind farm nodes, boundary nodes, all the nodes and all the lines in subsystem $j$, respectively. In power balance constraints, define phase angle $\theta_{n}=0$ when the corresponding bus is the slack bus. Note that there is just one slack bus for all the subsystems.

3) Operation constraints: The linearized branch flow limits (23)-(26), and voltage and frequency constraints (27) and (28). Constraint (28) is a linear frequency limit considering the total capacity of restored generators $s$ and their frequency response constant $\varepsilon$ [15]. Inequalities (12) and (28) are the reserve constraints in the restoration process considering the $N-1$ security criterion of generators. Note that the original quadratic constraint of branch flow $-S_{n m, \text { max }}^{2} \leq P_{n m}^{2}+Q_{n m}^{2} \leq S_{n m, \text { max }}^{2}$ is linearized by (23)-(26).

$$
\begin{array}{r}
-S_{n m, \text { max }} \leq P_{n m} \leq S_{n m, \text { max }} \\
-S_{n m, \text { max }} \leq Q_{n m} \leq S_{n m, \text { max }} \\
-\sqrt{2} S_{n m, \text { max }} \leq P_{n m}+Q_{n m} \leq \sqrt{2} S_{n m, \text { max }} \\
-\sqrt{2} S_{n m, \text { max }} \leq P_{n m}-Q_{n m} \leq \sqrt{2} S_{n m, \text { max }} \\
V_{n, \text { min }}<V_{n}<V_{n, \text { max }} \quad \forall n \in N_{S u b_{j}} \\
\Delta f=\mid \sum_{i \in N_{\mathrm{L}, j}} x_{\mathrm{L}, i} p_{\mathrm{L}, i}-p_{\mathrm{L}}^{0}-\sum_{C_{i} \in S u b_{j}} p_{\mathrm{B}, j, C_{i}} \\
+p_{\mathrm{B}}^{0}-\sum_{i \in N_{\mathrm{W}, j}} p_{\mathrm{W}, i}+p_{\mathrm{W}}^{0}\left|/ \sum_{i^{e} \in N_{\mathrm{G}, j,}, i^{e} \neq i} \frac{s_{i^{e}}}{\varepsilon_{i}}\right| \leq \max \Delta f
\end{array}
$$

\section{B. Linearization of robust distributed models}

The distributed subsystem models are coordinated by the coordinator using Model (29) which is corresponding to (5)(8).

$$
\begin{aligned}
& \operatorname{Min}_{\boldsymbol{S}_{C_{i}}} \sum_{C_{i} \in N_{\text {tie }}}\left[\left(\boldsymbol{S}_{C_{i}}-\boldsymbol{S}_{\mathrm{B}, C_{i}}^{*}\right) \boldsymbol{w}_{C_{i}}\left(\boldsymbol{S}_{C_{i}}-\boldsymbol{S}_{\mathrm{B}, C_{i}}^{*}\right)^{\mathrm{T}}+\boldsymbol{v}_{C_{i}}\left(\boldsymbol{S}_{C_{i}}-\boldsymbol{S}_{\mathrm{B}, C_{i}}^{*}\right)\right] \\
& \text { s.t. } \boldsymbol{S}_{C_{i}}=\left[\boldsymbol{S}_{C_{i}, j}, \boldsymbol{S}_{C_{i}, k}\right]=\left[p_{C_{i}, j}, \theta_{C_{i}, j}, p_{C_{i}, k}, \theta_{C_{i}, k}\right]
\end{aligned}
$$

(6) $-(8)$

Model (29) has linearized constraints and a quadratic objective function. As shown in Fig. 3, by introducing binary variables $u$, the objective function can be transformed into piecewise linearization functions (30) with constraints (31) [25]. $N_{C i}$ is the set of the boundary node of tie-line $C_{i}$. Finally, the coordinator model becomes the MILP form in (32) where $\boldsymbol{U}_{C i, i}, \boldsymbol{S}_{C, i}$, $\boldsymbol{O}_{C i, i}$ and $\boldsymbol{O}_{C, i}^{\prime}$ are the set of $u_{C i, i, o}, s_{C i, i, o}, O_{C i, i, o}$ and $O_{C i, i, o+1}$, respectively.

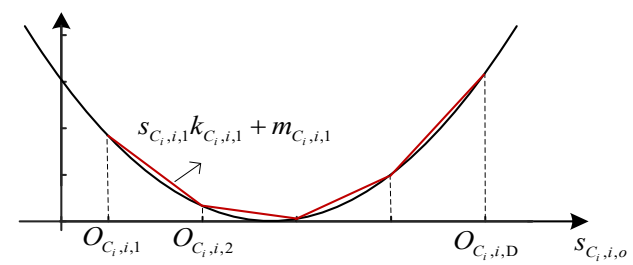

Fig. 3. Diagram of the piecewise linearization

$$
\begin{aligned}
& \sum_{C_{i} \in N_{\mathrm{tie}}} \sum_{i \in N_{C_{i}}} \sum_{o=1}^{D-1} w_{C_{i}, i}\left(s_{C_{i}, i, o} k_{C_{i}, i, o}+m_{C_{i}, i, o} u_{C_{i}, i, o}\right)+\sum_{C_{i} \in N_{\mathrm{tie}}} \boldsymbol{v}_{C_{i}} \boldsymbol{S}_{C_{i}} \\
& \text { s.t. } \sum_{o=1}^{D-1} u_{C_{i}, i, o}=1 \\
& \sum_{o=1}^{D-1} s_{C_{i}, i, o}=s_{C_{i}, i} \\
& O_{C_{i}, i, o} u_{C_{i}, i, o} \leq s_{C_{i}, i, o} \leq O_{C_{i}, i, o+1} u_{C_{i}, i, o}
\end{aligned}
$$




\section{The coordinator model}

$$
\begin{aligned}
& \underset{\boldsymbol{s}_{C_{i}}}{\operatorname{Min}}\left[\sum_{C_{i} \in N_{\mathrm{tic}}} \boldsymbol{w}_{C_{i}}\left(\boldsymbol{S}_{C_{i}} \boldsymbol{K}_{C_{i}}^{\mathrm{T}}+\boldsymbol{M}_{C_{i}} \boldsymbol{U}_{C_{i}}^{\mathrm{T}}\right)+\sum_{C_{i} \in N_{\mathrm{tie}}} \boldsymbol{v}_{C_{i}} \boldsymbol{S}_{C_{i}}^{\mathrm{T}}\right] \\
& \text { s.t. }\left\{\begin{array}{l}
\boldsymbol{S}_{C_{i}}=\left[\operatorname{sum}\left(\boldsymbol{S}_{C_{i}, 1}^{\prime}\right), \ldots, \operatorname{sum}\left(\boldsymbol{S}_{C_{i}, i}^{\prime}\right), \ldots\right] \\
\operatorname{sum}\left(\boldsymbol{U}_{C_{i}, i}\right)=1 \\
\operatorname{sum}\left(\boldsymbol{S}_{C_{i}, i}^{\prime}\right)=\boldsymbol{S}_{C_{i}, i} \\
\boldsymbol{O}_{C_{i}, i} \boldsymbol{U}_{C_{i}, i}^{\mathrm{T}} \leq \boldsymbol{S}_{C_{i}, i}^{\mathrm{T}} \leq \boldsymbol{O}_{C_{i}, i}^{\prime} \boldsymbol{U}_{C_{i}, i}^{\mathrm{T}} \\
(6)-(11) \quad\left(\forall i \in N_{C_{i}}, C_{i} \in N_{\text {tie }}\right)
\end{array}\right.
\end{aligned}
$$

The subsystem model is a two-stage robust optimization in (33). The first stage addresses load pickup decisions and the second stage handles recourse decisions to reveal the uncertainty [26]. With the Min-Max-Min form of the objective function, the optimal solution maximizes load pickup amount and minimizes the boundary mismatch in the worst-case. $\boldsymbol{S}_{\mathrm{C}, j}^{*}$ is the set of $p_{C_{i, j}}^{*}$ and $\theta_{C_{i, j}}^{*}$.

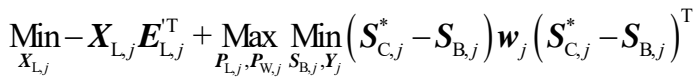

$$
\begin{aligned}
& +\boldsymbol{v}_{j}\left(\boldsymbol{S}_{\mathrm{C}, j}^{*}-S_{\mathrm{B}, j}\right) \\
& \text { s.t } \Delta=\left\{\boldsymbol{X}_{\mathrm{L}, j}\right\} \text {, } \\
& \Psi=\left\{\begin{array}{l}
\left(\boldsymbol{S}_{\mathrm{B}, j}, \boldsymbol{Y}_{j}\right):(11)-(28) \\
\boldsymbol{Y}_{j}=\left(p_{\mathrm{G}, i}, p_{n}, q_{n}, P_{n m}, Q_{n m}, \delta_{n}, \theta_{n}, \operatorname{app}\left(\cos \theta_{n m}\right)\right) \\
i \in N_{G, j}, n \in N_{\mathrm{Subj}}, C_{i} \in S u b_{j}
\end{array}\right\} \\
& \Phi=\left\{\left(\boldsymbol{P}_{\mathrm{L}, j}, \boldsymbol{P}_{\mathrm{W}, j}\right): p_{l o w, i} \leq p_{i} \leq p_{u p, i}, i \in N_{\mathrm{L}, j} \& N_{\mathrm{W}, j}\right\}
\end{aligned}
$$

Because all the constraints in (33) are linear, the inner-level Max-Min problem is converted into a compact form with linearized equality and inequality (34).

$$
\begin{aligned}
& \underset{\left(\boldsymbol{P}_{\mathrm{L}, j}, \boldsymbol{P}_{\mathrm{W}, j}\right) \in \Phi}{\operatorname{Max}} \underset{\boldsymbol{S}_{\mathrm{B}, j}, \boldsymbol{Y}_{j}}{\operatorname{Min}}\left(\boldsymbol{S}_{\mathrm{C}, j}^{*}-\boldsymbol{S}_{\mathrm{B}, j}\right) \boldsymbol{w}_{j}\left(\boldsymbol{S}_{\mathrm{C}, j}^{*}-\boldsymbol{S}_{\mathrm{B}, j}\right)^{\mathrm{T}}+\boldsymbol{v}_{j}\left(\boldsymbol{S}_{\mathrm{C}, j}^{*}-\boldsymbol{S}_{\mathrm{B}, j}\right) \\
& \text { s.t. } \boldsymbol{A} \boldsymbol{S}_{\mathrm{B}, j}+\boldsymbol{B} \boldsymbol{Y}_{j}+\boldsymbol{D} \boldsymbol{X}_{\mathrm{L}, j} \boldsymbol{P}_{\mathrm{L}, j}+\boldsymbol{E} \boldsymbol{P}_{\mathrm{W}, j}+\boldsymbol{F}=0 \\
& \quad \boldsymbol{G}_{\mathrm{B}, j}+\boldsymbol{H} \boldsymbol{Y}_{j}+\boldsymbol{I} \leq 0
\end{aligned}
$$

When load pickup decision variables in $\boldsymbol{X}_{\mathrm{L}, j}$ are determined in the first stage, the Min problem in (34) is a convex optimization with a quadratic objective function and linear constraints. By applying the KKT condition, the solution of the Min problem can be obtained [27]. Then, Model (34) is further transformed into Model (35).

$$
\begin{aligned}
& \underset{\left(P_{L, j}, P_{W, j}\right) \in \Phi}{\operatorname{aax}}\left(\boldsymbol{S}_{\mathrm{B}, j}-\boldsymbol{S}_{\mathrm{C}, j}^{*}\right) \boldsymbol{w}_{j}\left(\boldsymbol{S}_{\mathrm{B}, j}-\boldsymbol{S}_{\mathrm{C}, j}^{*}\right)^{\mathrm{T}} \\
& \quad+\boldsymbol{v}_{j}\left(\boldsymbol{S}_{\mathrm{B}, j}-\boldsymbol{S}_{\mathrm{C}, j}^{*}\right) \\
& \text { s.t }\left\{\begin{array}{l}
2 \boldsymbol{Q} \boldsymbol{S}_{\mathrm{B}, j}{ }^{\mathrm{T}}+\boldsymbol{C}+\boldsymbol{\lambda}^{\mathrm{T}} \boldsymbol{A}+\boldsymbol{\mu}^{\mathrm{T}} \boldsymbol{G}=0 \\
\boldsymbol{\lambda}^{\mathrm{T}} \boldsymbol{B}+\boldsymbol{\mu}^{\mathrm{T}} \boldsymbol{H}=0 \\
\boldsymbol{A} \boldsymbol{S}_{\mathrm{B}, j}+\boldsymbol{B} \boldsymbol{Y}_{j}+\boldsymbol{D} \boldsymbol{X}_{\mathrm{L}, j}^{*} \boldsymbol{P}_{\mathrm{L}, j}+\boldsymbol{E} \boldsymbol{P}_{\mathrm{W}, j}+\boldsymbol{F}=0 \\
\boldsymbol{G} \boldsymbol{S}_{\mathrm{B}, j}+\boldsymbol{H} \boldsymbol{Y}_{j}+\boldsymbol{I} \leq 0 \\
\boldsymbol{\mu}^{\mathrm{T}}\left(\boldsymbol{G} \boldsymbol{S}_{\mathrm{B}, j}+\boldsymbol{H} \boldsymbol{Y}_{j}+\boldsymbol{I}\right)=0 \\
\boldsymbol{\mu}^{\mathrm{T}} \geq 0
\end{array}\right. \\
& \left\{\begin{array}{l}
0 \leq \boldsymbol{\mu} \leq \mathrm{M} \boldsymbol{Z} \\
\mathrm{M}(\boldsymbol{Z}-\boldsymbol{I}) \leq \boldsymbol{G} \boldsymbol{S}_{\mathrm{B}, j}+\boldsymbol{H} \boldsymbol{Y}_{j}+\boldsymbol{I} \leq 0
\end{array}\right.
\end{aligned}
$$

\section{The subsystem model}

$$
\begin{aligned}
& \underset{\boldsymbol{X}_{\mathrm{L}, j}}{\operatorname{Min}}-\boldsymbol{X}_{\mathrm{L}, j} \boldsymbol{E}_{\mathrm{L}, j}^{\mathrm{T}}+\underset{\boldsymbol{S}_{\mathrm{B}, j}, \boldsymbol{Y}_{j}, \boldsymbol{P}_{\mathrm{L}, j}, \boldsymbol{P}_{\mathrm{W}, j}}{\operatorname{Max}}\left[\boldsymbol{w}_{j}\left(\boldsymbol{S}_{\mathrm{B}, j} \boldsymbol{K}_{\mathrm{B}, j}^{\mathrm{T}}+\boldsymbol{M}_{\mathrm{B}, j} \boldsymbol{U}_{\mathrm{B}, j}^{\mathrm{T}}\right)+\boldsymbol{v}_{j} \boldsymbol{S}_{\mathrm{B}, j}^{\mathrm{T}}\right] \\
& \text { s.t. } \Delta=\left\{\boldsymbol{X}_{\mathrm{L}, j}:(11)\right\}
\end{aligned}
$$

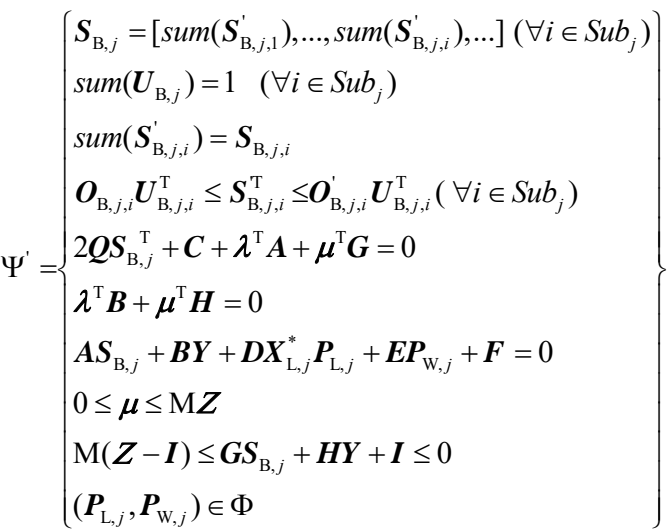

The last three constraints in (35) are the complementary slackness condition which can be further linearized. Introducing binary variables in $\mathbf{Z}$ and the constant $M$ with a sufficient large value linearizes the complementarity slackness condition as constraints (36). However, it adds binary variables and makes (35) become an MIQP model. The quadratic term in the objective function is transformed into (37) with the same piecewise linearization method of the coordinator model (32). Accordingly, the inner-level Max-Min optimization becomes an MILP problem (37).

By now, the robust distributed restoration models for the coordinator (32) and subsystems (37) are all converted into MILP form and are solved by an iterative parallel calculation process shown in the next section.

\section{Solution Method of Robust Distributed Models}

This section develops an IOUL algorithm to solve the robust distributed models in Section III. The inner-out (IO) iteration achieves independent restoration decision-making of regional grids by solving the subsystem problems in parallel. The global coordination is achieved by the upper-lower (UL) iteration between the coordinator and subsystem models. Through tie-line information exchange, the coordinated parallel restoration strategy is obtained according to convergence.

A. Solving coordinator-subsystem model by IOUL algorithm

With all the models in MILP forms, the solving process using the IOUL algorithm is shown as follows. Note that the lower level loop Steps 1 - 4 are for each subsystem and are performed in parallel.

Step 0: Set $L_{\text {upp }}=1$, upper-lower convergence thresholds $\varepsilon_{2} \geq$ 0 and $\varepsilon_{3} \geq 0$. Choose the initial values of shared variables in $S$ ${ }_{\mathrm{C}, j}^{\text {Lupp }}$ for each subsystem, and define the initial values of the penalty multipliers in $\boldsymbol{v}^{L_{u p p}}$ and $\boldsymbol{w}^{L_{u p p}}$.

Step 1: Set $L B=-\infty, U B=+\infty$, the inner-outer tolerance $\varepsilon_{1}$ $\geq 0, L_{\text {low }}=1$ and arbitrarily select a feasible $\boldsymbol{X}_{\mathrm{L}, j}^{\mathrm{Llow}} \in \Delta, \boldsymbol{X}_{\mathrm{L}, j}^{*}=\boldsymbol{X}$ Llow.

Step 2: Solve the inner-level problem (38) of the subsystem model (37) and obtain the worst-case wind output $\boldsymbol{P}_{\mathrm{w}, j}^{*}$ and load 
amount $\boldsymbol{P}_{\mathrm{L}, j}^{*}$ Update $U B=\min \left\{U B, o b j_{\mathrm{in}}+\boldsymbol{X}_{\mathrm{L}, j}^{L l o w}\right\}$ where $o b j_{\text {in }}$ is the objective function value of (38) in the $L_{\text {low }}$-th iteration.

$$
\begin{array}{ll}
\boldsymbol{s}_{\mathrm{B}, j}, \boldsymbol{Y}_{j}, \boldsymbol{P}_{\mathrm{L}, j}, \boldsymbol{P}_{\mathrm{W}, j} \\
\text { s.t. } & \boldsymbol{S}_{\mathrm{B}, j}=\left[\operatorname{sum}\left(\boldsymbol{S}_{\mathrm{B}, j, 1}^{\prime}\right), \ldots, \operatorname{sum}\left(\boldsymbol{S}_{\mathrm{B}, j, i}^{\prime}\right), \ldots\right]\left(\forall i \in \operatorname{Su} b_{j}\right) \\
& \operatorname{sum}\left(\boldsymbol{U}_{\mathrm{B}, j}^{\mathrm{T}}\right)=1 \quad\left(\forall i \in \operatorname{Sub} b_{j}\right) \\
& \operatorname{sum}\left(\boldsymbol{S}_{\mathrm{B}, j, i}^{\prime}\right)=\boldsymbol{S}_{\mathrm{B}, j, i} \\
& \boldsymbol{O}_{\mathrm{B}, j, i} \boldsymbol{U}_{\mathrm{B}, j, i}^{\mathrm{T}} \leq \boldsymbol{S}_{\mathrm{B}, j, i}^{\mathrm{T}} \leq \boldsymbol{O}_{\mathrm{B}, j, i}^{\prime} \boldsymbol{U}_{\mathrm{B}, j, i}^{\mathrm{T}}\left(\forall i \in S u b_{j}\right) \\
& 2 \boldsymbol{Q} \boldsymbol{S}_{\mathrm{B}, j}^{\mathrm{T}}+\boldsymbol{C}+\boldsymbol{\lambda}^{\mathrm{T}} \boldsymbol{A}+\boldsymbol{\mu}^{\mathrm{T}} \boldsymbol{G}=0 \\
& \lambda^{\mathrm{T}} \boldsymbol{B}+\boldsymbol{\mu}^{\mathrm{T}} \boldsymbol{H}=0 \\
& \boldsymbol{A} \boldsymbol{S}_{\mathrm{B}, j}+\boldsymbol{B} \boldsymbol{Y}_{j}+\boldsymbol{D} \boldsymbol{X}_{\mathrm{L}, j}^{*} \boldsymbol{P}_{\mathrm{L}, j}^{\mathrm{T}}+\boldsymbol{E} \boldsymbol{P}_{\mathrm{W}, j}+\boldsymbol{F}=0 \\
& 0 \leq \boldsymbol{\mu} \leq \mathrm{M} \boldsymbol{Z} \\
& \mathrm{M}(\boldsymbol{Z}-\boldsymbol{I}) \leq \boldsymbol{G} \boldsymbol{S}_{\mathrm{B}, j}+\boldsymbol{H} \boldsymbol{Y}_{j}+\boldsymbol{I} \leq 0 \\
& \left(\boldsymbol{P}_{\mathrm{L}, j}, \boldsymbol{P}_{\mathrm{W}, j}\right) \in \Phi
\end{array}
$$

Step 3: Solve the outer-level model (39) and obtain the optimal result $\boldsymbol{X}_{\mathrm{L}, j}^{l}\left(l \in\left\{1, \cdots, L_{\text {low }}\right\}\right)$, let $\boldsymbol{X}_{\mathrm{L}, j}^{L \text { Low }}=\boldsymbol{X}_{\mathrm{L}, j}^{*}$. Update $L B=\max \left\{L B, o b j_{\text {out }}\right\}$ where $o b j_{\text {out }}$ is the objective function value of (39) in the $L_{\text {low }}$ th iteration.

$$
\begin{array}{ll}
\vartheta_{j, j}, X_{\mathrm{L}, j}, \boldsymbol{S}_{\mathrm{B}, j}^{\prime}, \mathbf{Y}^{\prime} \\
\text { s.t. } & \boldsymbol{X}_{\mathrm{L}, j} \in \Delta \\
& \vartheta_{j} \geq \boldsymbol{W}_{\mathrm{L}, j}\left(\boldsymbol{E}_{\mathrm{L}, j}^{\mathrm{T}}+\vartheta_{\mathrm{B}, j} \boldsymbol{K}_{\mathrm{B}, j}^{\mathrm{T}}+\boldsymbol{M}_{\mathrm{B}, j} \boldsymbol{U}_{\mathrm{B}, j}^{\mathrm{T}}\right)+\boldsymbol{v}_{j} \boldsymbol{S}_{\mathrm{B}, j}^{\mathrm{T}} \\
& \boldsymbol{S}_{\mathrm{B}, j}^{l}=\left[\operatorname{sum}\left(\boldsymbol{S}_{\mathrm{B}, j, 1}^{\prime l}\right), \ldots, \operatorname{sum}\left(\boldsymbol{S}_{\mathrm{B}, j, i}^{l}\right), \ldots\right]\left(\forall i \in \operatorname{Sub} b_{j}\right) \\
& \operatorname{sum}\left(\boldsymbol{U}_{\mathrm{B}, j, i}^{l l_{w}}\right)=1 \quad\left(\forall i \in \operatorname{Sub} b_{j}\right) \\
& \boldsymbol{O}_{\mathrm{B}, j, i} \boldsymbol{U}_{\mathrm{B}, j, i}^{l \mathrm{~T}} \leq \boldsymbol{S}_{\mathrm{B}, j i}^{\prime \mathrm{T}} \leq \boldsymbol{O}_{\mathrm{B}, j, i}^{\prime} \boldsymbol{U}_{\mathrm{B}, j, i}^{l \mathrm{~T}}\left(\forall i \in \operatorname{Sub} b_{j}\right) \\
& \boldsymbol{A} \boldsymbol{S}_{\mathrm{B}, j}^{l}+\boldsymbol{B} \boldsymbol{Y}_{j}^{l}+\boldsymbol{D} \boldsymbol{X}_{\mathrm{L}, j} \boldsymbol{P}_{\mathrm{L}, j}^{*}+\boldsymbol{E} \boldsymbol{P}_{\mathrm{W}, j}^{*}+\boldsymbol{F}=0 \\
& \boldsymbol{G} \boldsymbol{S}_{\mathrm{B}, j}^{l}+\boldsymbol{H} \boldsymbol{Y}_{j}^{l}+\boldsymbol{I} \leq 0 \\
& \forall l \in\left\{1,2 \ldots L_{l o w}\right\}
\end{array}
$$

Step 4: If $U B-L B \leq \varepsilon_{1}$, obtain the result $\boldsymbol{S}_{\mathrm{B}, j}^{L \text { Low }}$ and go to Step 5 . Otherwise, $L_{\text {low }}=L_{\text {low }}+1$ and go to Step 2 .

Step 5: Collect $\boldsymbol{S}_{\mathrm{B}, j}^{L_{\text {Low }}}$ from the lower level $\boldsymbol{S}_{\mathrm{B}}^{L_{\text {Lup }}}=\left[\ldots, \boldsymbol{S}_{\mathrm{B}, j}^{L_{\text {Low }}}, \ldots\right]$. Solve the coordinator model (32) with $\boldsymbol{S}_{\mathrm{B}}^{*}=\boldsymbol{S}_{\mathrm{B}}^{L \text { Lwp }}$. Obtain $\boldsymbol{S}_{\mathrm{C}}^{L_{\text {wp }}+1}$.

Step 6: Check convergence by the necessary consistency equations (40) and sufficient stopping criteria (41). If they are not satisfied and the iteration limit (42) is not reached, proceed to Step 7. Otherwise, stop and obtain the final result.

$$
\begin{gathered}
\mid \boldsymbol{S}_{\mathrm{B}, j}^{L_{u p p}-S_{\mathrm{C}, j}^{L_{u p p}} \mid \leq \boldsymbol{\varepsilon}_{2} \quad} \quad \forall j \in N_{\mathrm{Sub}} \\
\left|\left(f_{j}^{L_{u p p}}-f_{j}^{L_{u p p}-1}\right) / f_{j}^{L_{u p p}}\right| \leq \varepsilon_{3} \quad \forall j \in N_{\mathrm{Sub}} \\
L_{u p p} \geq L_{\max }
\end{gathered}
$$

Step 7: Update penalty parameters $v^{L_{\text {wpp }}}$ and $w^{L_{\text {Lqp }}}$ according to (43) and (44). Set $L_{u p p}=L_{u p p}+1$ and go to Step 1 .

$$
\begin{aligned}
& \boldsymbol{v}_{j}^{L_{\text {qpp }}+1}=\boldsymbol{v}_{j}^{L_{\text {} q p}}+2\left(\boldsymbol{w}_{j}^{L_{\text {qp }}}\right) Q\left(\boldsymbol{w}_{j}^{L_{\text {qp }}}\right)^{\mathrm{T}}\left(\boldsymbol{S}_{\mathrm{B}, j}^{L_{\text {qp }}}-\boldsymbol{S}_{\mathrm{C}, j}^{L_{q p}}\right) \quad \forall j \in N_{\text {Sub }} \\
& \boldsymbol{w}_{j}^{L_{\text {upp }}+1}=\boldsymbol{\beta} \boldsymbol{w}_{j}^{L_{\text {upp }}}(\beta \geq 1) \quad \forall j \in N_{\mathrm{Sub}}
\end{aligned}
$$

The algorithm starts with an inner-outer iterative calculation (Step 1-Step 4) according to the column-and-constraint generation (C\&CG) method [28] which is proven to converge for the MILP problem [22], [26]. The whole IO loop belongs to the lower level of an upper-lower iteration which is based on the augmented Lagrangian ATC method [18] with good convergence characteristics, even for non-convex models [13], [19]. The detailed convergence characteristic of the ATC method is provided in [29]. Therefore, the IOUL algorithm generally converges for the robust distributed restoration model.

\section{B. Coordination process in iteration of IOUL algorithm}

Fig. 4 shows the structure of the whole IOUL loop and the boundary information exchange process. The IO loop (Step 1Step 4) solves the subsystem model (37) in parallel. The inner optimization (Step 2) returns the worst-case conditions $\left(\boldsymbol{P}_{\mathrm{w}, \mathrm{w}}^{*}, \boldsymbol{P}\right.$ ${ }_{\mathrm{L}, j}^{*}$ ) to the outer optimization (Step 3), while the outer optimization determines the load pickup variables $\boldsymbol{X}_{\mathrm{L}}^{*}$ for the inner optimization. The whole IO loop belongs to the lower level of the UL loop. Results in $S_{\mathrm{B}, j}^{*}$ from the IO loop are delivered to the upper level optimization (Step 5) to solve the coordinator model (32). The final coordinated parallel restoration strategy is obtained when the IOUL algorithm converges.

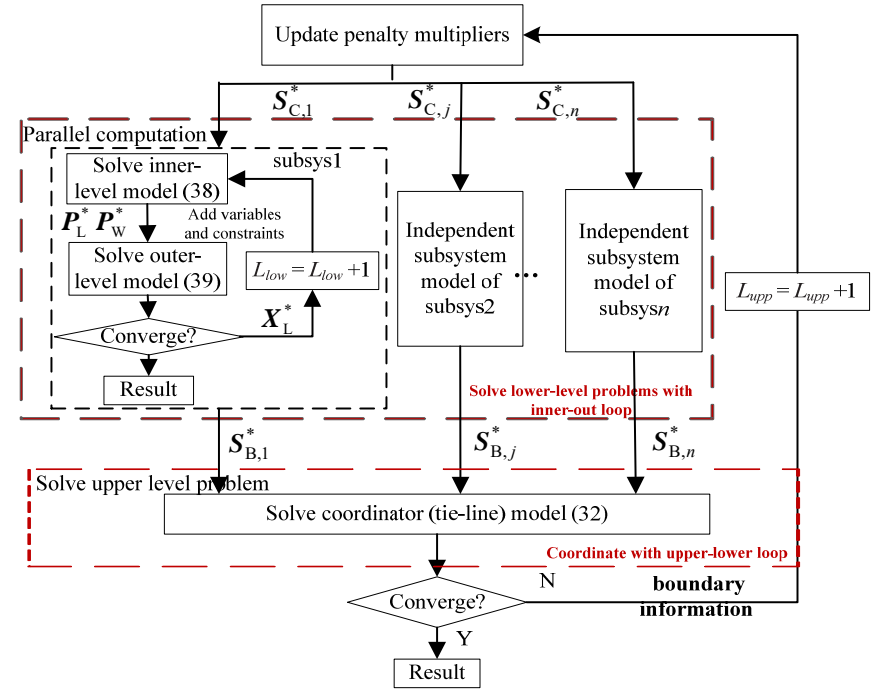

Fig. 4. Structure of the IOUL loop

The robust distributed coordination is achieved with the IOUL algorithm. The parallel restored subsystems maximize regional load pickup amount as well as minimize the worstcase of boundary variable mismatch. With the tie-line information, the coordinator coordinates these independent restoration strategies of subsystems to pursue a global restoration optimization. In this scheme, each subsystem makes restoration decisions independently and is coordinated by the boundary variable information exchange. The independent subsystem restoration strategy is the result of the IO loop convergence and the coordination is achieved when the UL loop converges. Even in the worst-case, the boundary mismatch of the robust distributed restoration strategy can be maintained within $\varepsilon_{2}$. That means the feasibility of the corresponding distributed coordination scheme can always be guaranteed in the uncertain condition.

\section{CASe Study}

A three-area 18-bus system [13] and a sectionalized IEEE118-bus system [30] are used to demonstrate the effectiveness of the proposed method. The convergence thresholds 
of the IOUL algorithm are $0.5 \%$. The initial values of boundary variables are set as 0 , and the initial penalty values in $\boldsymbol{v}$ and $w$ are set as 0 and 1, respectively. $\beta$ is set as $1.8 .10 \%$ and $15 \%$ deviations from the expected values are used to determine the bound of polyhedral uncertainty sets of load and wind power output, respectively. All case studies were conducted using GUROBI V7.5.1 on a computer with Intel(R) Core(TM) i78550U CPU and 16 GB RAM.

\section{A. Performance of IOUL algorithm in 18-bus system}

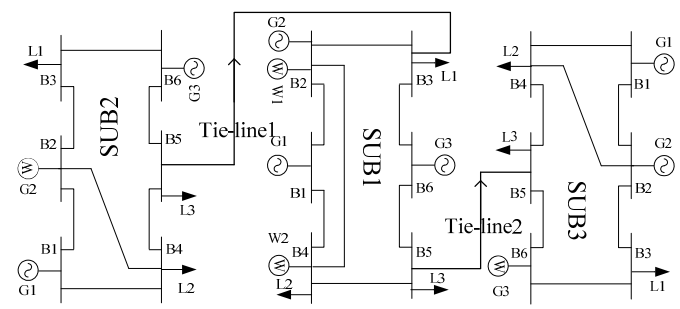

Fig. 5. Three-area 18-bus system

Fig. 5 shows the three-area 18-bus system and Fig. 6 presents the iteration process of obtaining the robust distributed coordination strategy. There are 6 UL loops. The IO loops are 26 for SUB1, 6 for SUB2 and 10 for SUB3, respectively. As shown in Fig. 6, boundary variables of SUB1 $p_{\mathrm{B}, 1, C 1}$ and $p_{\mathrm{B}, 1, C 2}$ are used in the IO loop and the result of the IO loop is used as the initial values for the UL loop. The Tie-line variables $p_{C 1,2}$ in Tie-line 1 and $p_{C 2,3}$ in Tie-line 2 are used in the UL loop. In the UL loop, the IO loop is implemented for each subsystem. The coordination among subsystems is realized by the UL loop. Finally, the largest difference of the power exchange between SUB1 and tie-line 1 is below $0.5 \%$, and so does the one between SUB1 and tie-line 2. Besides, the phase angle difference (Theta) is below 0.34 rad to avoid the SPA problem.

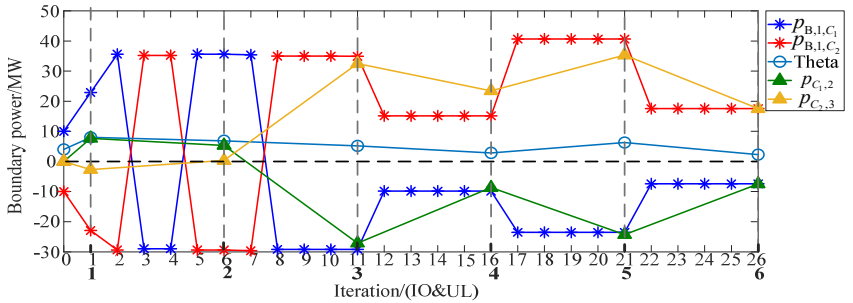

Fig. 6. IOUL iteration process of the distributed robust scheme

To demonstrate the advantages of the proposed method, the results are compared in Table I with the results of standard ATC [19] and centralized robust method [16]. In Table I, Objective $(\mathrm{MW})$ is the expected value of load recovery amount, while Objective (p.u.) is the value of objective function with the piecewise linear approximation of quadratic terms. The power exchange between SUB1 and SUB2 is $7.43 \mathrm{MW}$, and the one between SUB1 and SUB3 is $17.57 \mathrm{MW}$. It shows that using the proposed robust ATC method, the boundary mismatch is below the threshold $0.5 \%$. Under uncertain conditions, the result obtained with the standard ATC show an unacceptable $27.8 \%$ mismatch in the worst-case. Although more loads are restored, the standard ATC based coordination scheme is infeasible, because the unacceptable boundary power mismatch easily leads to security violation. The proposed method obtains the same objective value with the centralized robust optimization method [16]. This means that the proposed method can achieve global optimal coordination, although subsystems make independent restoration decisions. Overall, the proposed method maintains the feasibility of the distributed scheme under uncertain conditions and achieves coordination of the whole system.

TABLE I

COMPUTATION EFFICIENCY OF DIFFERENT METHOD

\begin{tabular}{ccccc}
\hline \hline & $\begin{array}{c}\text { Objective } \\
\text { (p.u.) }\end{array}$ & $(\mathrm{MW})$ & $\begin{array}{c}\text { Ther recovery } \\
\text { (MW) }\end{array}$ & $\begin{array}{c}\text { Boundary } \\
\text { mismatch }\end{array}$ \\
\hline IOUL & -2.41 & 250 & $\begin{array}{c}\text { Tie-line1: 7.43 } \\
\text { Tie-line2:17.57 }\end{array}$ & $0.3 \%$ \\
\hline ATC [19] & -2.94 & 300 & $\begin{array}{r}\text { Tie-line1: 66.5 } \\
\text { Tie-line2: } 77\end{array}$ & $27.8 \%$ \\
\hline Centralized [16] & -2.50 & 250 & $\begin{array}{c}\text { Tie-line1: } 35 \\
\text { Tie-line2: } 70\end{array}$ & $/$ \\
\hline \hline
\end{tabular}

B. Restoration performance in IEEE 118-bus system

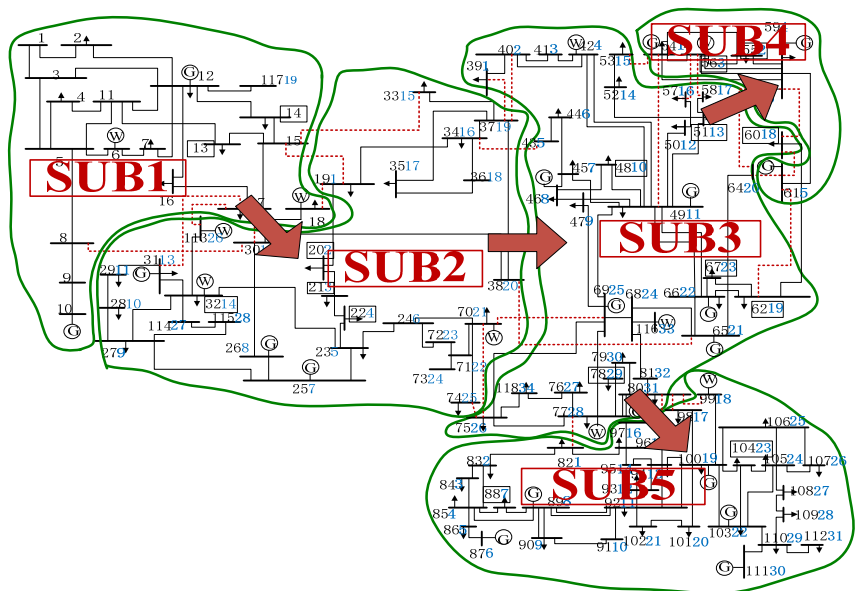

Fig. 7. IEEE-118 bus system with five subsystems

According to [30], the IEEE-118 bus system is divided into five subsystems which can balance power supply and demand by themselves. The computation efficiency of the IOUL method is compared with the centralized robust optimization in [19] which solves the whole system restoration model with the C\&CG method. The restoration performance of the proposed coordination scheme is compared with the restoration of five parallel restored subsystems in [30].

TABLE II

COMPARISONS OF DIFFERENT SCHEMES

\begin{tabular}{cccc}
\hline \hline & $\begin{array}{c}\text { IOUL based } \\
\text { Coordinated } \\
\text { scheme }\end{array}$ & $\begin{array}{c}\text { Separated } \\
\text { scheme [30] }\end{array}$ & $\begin{array}{c}\text { Centralized } \\
\text { scheme [16] }\end{array}$ \\
\hline Calculation time & $203.87 \mathrm{~s}$ & $17.24 \mathrm{~s}$ & $1057.96 \mathrm{~s}$ \\
\hline Load recovery & $3187 \mathrm{MW}$ & $3030 \mathrm{MW}$ & $3195 \mathrm{MW}$ \\
\hline Optimization gap & $0.25 \%$ & $5.44 \%$ & $/$ \\
\hline Restoration time & $30.44 \mathrm{~min}$ & $39.07 \mathrm{~min}$ & $/$ \\
\hline \hline
\end{tabular}

As shown in Table II, compared with the centralized robust optimization method, the proposed method reduces the calculation time from $1057.96 \mathrm{~s}$ to $203.87 \mathrm{~s}$ with an acceptable optimization gap. This is because the proposed method performs parallel optimization; thus, the recorded time of the lower iteration is the most time consuming subsystem IO loop calculation. The IO loop is based on the C\&CG algorithm. Since the distributed coordination scheme reduces the calculation size of the subsystem robust restoration optimization, the additional variables and constraints from the C\&CG algorithm are largely reduced, which further reduces the calculation time. Compared with the separated scheme, the coordinated parallel restoration scheme can restore all the loads in $30.44 \mathrm{~min}$ with 5 
steps, while the separated one picks up $96.25 \%$ loads in 39.07 min with 7 steps.

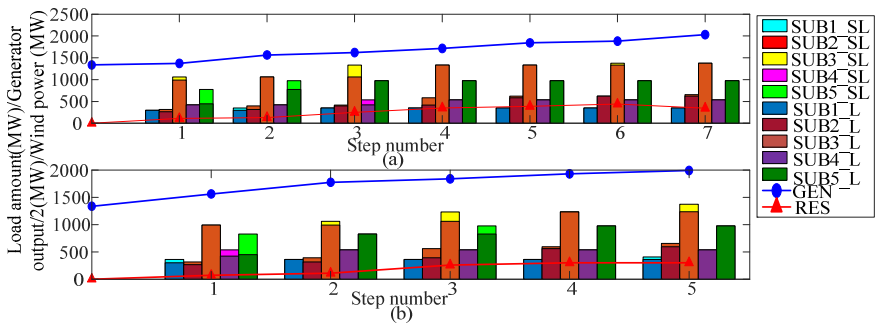

Fig. 8. Multi-step restoration conditions in the subsystem separated scheme (a) and coordinated scheme (b).

With the load pickup amount 'SUB_L', generator 'Gen' and wind power output 'RES', Fig. 8 demonstrates the 5 -step coordinated parallel restoration process and the 7-step separated restoration process. When the separated scheme chooses to restore the loads in their own area as 'SUB3_SL' in Step1 in Fig. 8 (a), the coordination scheme makes SUB3 support its neighboring grids first. As shown in Fig. 8 (b), although no load restored in SUB3, the generators in SUB3 still ramp up to support SUB2, SUB4 and SUB 5.

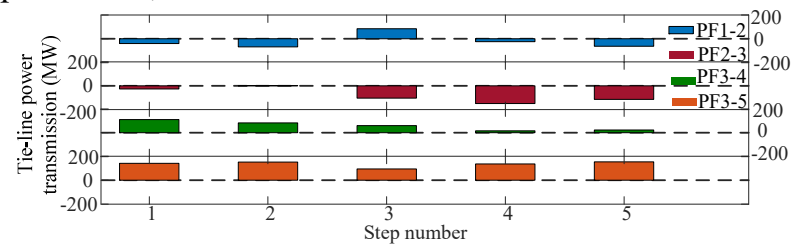

Fig. 9. Power exchange between subsystems in the coordinated scheme

Coordination of the whole system is achieved by the optimized power exchange among subsystems. Fig. 9 presents the optimized power exchange of four tie-lines in five restoration steps. Fig. 10 shows the voltage and phase angle at the boundary buses of subsystems. Besides, the boundary voltage and phase angle differences between neighboring subsystems are presented with 'distance' in (a) and (b), respectively. All the phase angle differences are below the required $0.34 \mathrm{rad}(19.48$ degree) to avoid the SPA problem. The boundary voltage values are obtained when reactive power of corresponding buses is regarded as zeros. The local voltage regulation can be performed to adjust boundary voltages if the voltage difference requirement is not satisfied.

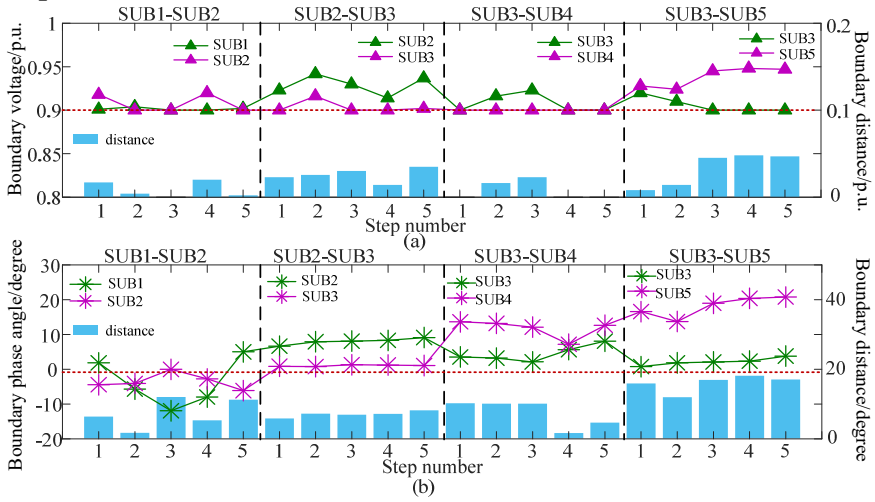

Fig. 10. Boundary voltage (a) and boundary phase angle (b) conditions of neighboring subsystems in the multi-step restoration process

Results in Fig. 9 correspond to the converged results in Fig. 10. Taking SUB1 and tie-line between SUB1 and SUB2 for example, Fig. 11 demonstrates the IOUL iteration number of each step. As can be seen, the whole process has 110 IO loops and 55 UL loops. The UL loop continues as long as the dis- tance of the inner and outer objective values of subsystem ' $U B-L B$ ' is below the threshold $\varepsilon_{1}$, and the step-by-step restoration strategies are obtained when the boundary variables $p_{\mathrm{B}, 1, C 1}$ in subsystem $1, p_{\mathrm{B}, 2, C 1}$ in subsystem 2 and $p_{C 1}$ in the tieline become consistent.

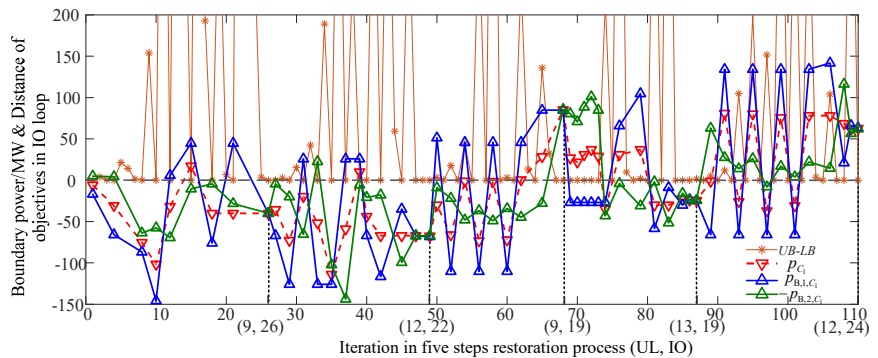

Fig. 11. Boundary variable iterations between SUB1 and SUB2

Note that the separated scheme cannot pick up the load block at Bus 18 in SUB1 using the robust optimization. This means that SUB1 has the risk of security violation to restore all the loads alone under the uncertain condition. In the coordinated scheme, the tie-line flow 'PF-2' in Step 5 in Fig. 9 is negative, which means SUB2 supplies power to SUB1, so that all the loads in SUB1 can be restored. Overall, the proposed scheme not only allows independent restoration of subsystems but also accelerates the restoration process and assists subsystems to handle the worst-condition under the uncertainty.

\section{DISCUSSION}

This paper uses a steady-state model to optimize load pickup and subsystem reconnection decision-making for the end of the PPSR stage. The frequency and voltage stability of subsystems can be controlled by the frequency response of generators and voltage regulation within individual subsystems considering boundary power exchanges. A linearized frequency constraint is incorporated into the subsystem model to ensure the frequency stability of the obtained result. For the voltage stability, the method in [31] can be applied to provide transient voltage constraints for each subsystem. Besides, extra simulations considering N-1 stability analysis can be performed before the implementation of the optimization result to maintain the stability in individual subsystems and during their coordinated restoration. More exact dynamic constraints for the frequency and voltage stabilities can be integrated into the model, and the proposed robust distributed method is feasible as long as these constraints are convex.

\section{CONCLUSION}

This paper presents a new coordination scheme for parallel restored subsystems in the build-up restoration for wind power penetrated transmission systems. The robust distributed optimization models are constructed with the coordinatorsubsystem framework. By applying the developed IOUL algorithm, a robust distributed coordination strategy is obtained by iteratively solving small-scale MILP models. The proposed scheme maintains the information privacy of subsystems by independent decision-making, coordinates the whole system restoration by boundary information interaction and provides robustness to handle the uncertain condition. The case study results show the computation efficiency benefits and good restoration performance of the proposed method. The robust distributed method ensures boundary consistency, even in the 
worst-case, and largely reduces the calculation burden of the robust restoration optimization without loss of optimality. The coordination of subsystems accelerates the restoration process and ensures complete regional grid restoration without security violation under uncertain conditions.

\section{REFERENCES}

[1] M. Adibi, P. Clelland, L. Fink, H. Happ, R. Kafka, J. Raine, D. Scheurer and F. Trefny, "Power system restoration- a task force report," IEEE Trans. Power Syst., vol. 2, no. 2, pp. 271-277, 1987.

[2] F. Qiu and P. Li, "An Integrated Approach for Power System Restoration Planning," Proc. IEEE, vol. 105, no. 7, pp. 1234-1252, 2017.

[3] N.A.Fountas, N.D. Hatziargyriou, C. Orfanogiannis and A. Tasoulis, "Interactive long-term simulation for power system restoration planning," IEEE Trans. Power Syst., vol, 12 no. 1, pp. 61 -68, 1997.

[4] C. Wang, V. Vittal, and K. Sun, "OBDD-based sectionalizing strategies for parallel power system restoration," IEEE Trans. Power Syst., vol. 26, no. 3, pp.1426-1433, 2011.

[5] S. Abbasi, M. Barati and G. J. Lim, "A parallel sectionalized restoration scheme for resilient smart grid systems," IEEE Trans. Smart Grid, vol. 10, no. 2, pp. 1660-1670, 2019.

[6] L. Sun, C. Peng, J. Hu and Y. Hou, "Application of type 3 wind turbines for system restoration," IEEE Trans. Power Syst., vol. 33, no. 3, pp. 3040-3051, 2018.

[7] Y. Zhao, Z. Lin, Y. Ding, Y. Liu, L. Sun and Y. Yan, "A model predictive control based generator start-up optimization strategy for restoration with microgrids as black-start resources," IEEE Trans. Power Syst., vol. 33, no. 6, pp. 7189-7203, 2018.

[8] K. L. Liou, C.C. Liu and R.F. Chu, "Tie line utilization during power system restoration," IEEE Trans. Power Syst., vol. 10, no. 1, pp. 192 199, 1995.

[9] A. Stefanov, C. C. Liu, M. Sforna, M. Eremia and R. Balaurescu, "Decision support for restoration of interconnected power systems using tie lines," IET Gener., Trans. \& Distr. Vol. 9, no. 11, pp. 1006-1018, 2015.

[10] Z. Lin, F. Wen, C. Chung, K. Pong and H. Zhou, "Division algorithm and interconnection strategy of restoration subsystems based on complex network theory," IET Gener., Trans. \& Distr. Vol. 5, no. 6, pp. 674-683, 2011.

[11] Continental Europe operation handbook. Policy 5: emergency operations' 2017, [Online]. Available: https://docstore.entsoe.eu/Documents/ Publications/SOC/Continental_Europe/oh/170926_Policy_5_ver3_1 43_RGCE_Plenary_approved.pdf

[12] C. Qi, K. Wang, Y. Fu, G. Li, B. Han, R. Huang and T. Pu. "A decentralized optimal operation of AC/DC hybrid distribution grids," IEEE Trans. Smart Grid, vol. 9, no. 6, pp. 6095-6105, 2018.

[13] M. Zhou, J. Zhai, G. Li and J. Ren, "Distributed dispatch approach for bulk AC/DC hybrid systems with high wind power penetration," IEEE Trans. Power Syst., vol. 33, no.3, pp. 3325-3336, 2018.

[14] S. Lei, J. Wang, C. Chen, and Y. Hou, "Mobile emergency generator prepositioning and real-time allocation for resilient response to natural disasters," IEEE Trans. Smart Grid, vol. 9, no. 3, pp. 2030-2041, 2018.

[15] A. Golshani, W. Sun, Q. Zhou, Q. P. Zheng and Y. Hou, "Incorporating wind energy in power system restoration planning," IEEE Trans. Smart Grid, vol. 10, no. 1, pp. 16-28, 2019.

[16] A. Golshani, W. Sun, Q. Zhou, Q. P. Zheng, J. Wong and F. Qiu, "Coordination of wind farm and pumped-storage hydro for a self-healing power grid," IEEE Trans. Sustain. energy, vol. 9, no. 4, pp. 1910-1920, 2018.

[17] G. Monique and S. Kim, "Lagrangean decomposition: A model yielding stronger Lagrangean bounds," Math. Program., vol. 39, no. 2, pp. 215228, 1987.

[18] S. Tosserams, L. F. P. Etman, P. Y. Papalambros, and J. E. Rooda, "An augmented Lagrangian relaxation for analytical target cascading using the alternating directions method of multipliers," Struct. Multdisc. Optim., vol. 31, no. 3, pp. 176-189, 2006.

[19] A. Kargarian, Y. Fu and Z. Li, "Distributed security-constrained unit commitment for large-scale power systems," IEEE Trans. Power Syst., vol. 30, no. 4, pp. 1925-1936, 2015.

[20] J. Zhao, H. Wang, Y. Liu, Q. Wu, Z. Wang and Y. Liu, "Coordinated restoration of transmission and distribution system using decentralized scheme," IEEE Trans. Power Syst., in press.
[21] S. Wunderlich, M. M. Adibi, R. Fischl and C. O. D. Nwankpa, "An approach to stand phase angle reduction," IEEE Trans. Power Syst., vol. 9, no. 1, pp. 470-478, 1994.

[22] L. Zhao and B. Zeng, "Robust unit commitment problem with demand response and wind energy," IEEE PES Generl Meeting, pp. 1-8, 2012.

[23] W. Sun, C.-C. Liu, and L. Zhang, "Optimal start-up strategy for bulk power system restoration," IEEE Trans. Power Syst., vol. 26, no. 3, pp. 1357-1366, Aug. 2011

[24] J. Zhao, H. Wang, Y. Liu, R. Azizipanah-Abarghooee and V. Terzija, "Utility-oriented on-line load restoration considering wind power penetration," IEEE Trans. Sustain. Energy, vol. 10, no. 2, pp. 706-717, 2019.

[25] M. Q. Wang, H. B. Gooi, S. Chen, and S. Lu, "A mixed integer quadratic programming for dynamic economic dispatch with valve point effect," IEEE Trans. Power Syst., vol. 29, no. 5, pp. 1-10, 2014.

[26] B. Zeng and L. Zhao, "Solving two-stage robust optimization problems using a column-and-constraint generation method," Oper. Res. Let., vol.41, no. 55, 2013

[27] D. P. Bertsekas, Nonlinear Programming, 2nd ed. Belmont, MA, USA Athena Scientific, 2003.

[28] S. Lei, C. Chen, H. Zhou, and Y. Hou, "Routing and scheduling of mobile power sources for distribution system resilience enhancement," IEEE Trans. Smart Grid, vol. 10, no. 5, pp. 5650-5662, 2019.

[29] N. F. Michelena, H. Park, and P. Y. Papalambros, "Convergence properties of analytical target cascading," AIAA J., vol. 41, no. 5, pp. 897-905, May 2003

[30] W. Liu, Z. Lin, F. Wen, C. Y. Chung, Y. Xue, G. Ledwich, "Sectionalizing strategies for minimizing outage durations of critical loads in parallel power system restoration with bi-level programming," Int. J. Electr. Power Energy Syst, 71, 327-334, 2015.

[31] H. Qu, Y. Liu, "Maximizing restorable load amount for specific substation during system restoration," Int. J. Elect. Power Energy Syst., vol. 43, no. 1, pp. 1213-1220, Dec. 2012.

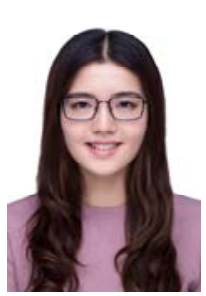

Jin Zhao (S'17) received the B.Sc. degree in electrical engineering in 2015 from Shandong University, Jinan, China, where she is working toward the Ph.D. degree in electrical engineering.

She is currently a visiting student at the Department of Electrical Engineering, Technical University of Denmark (DTU). Her research interests include power system resilience, restoration, renewable energy integration, and power system optimization.

Hongtao Wang (M'04-SM'15) received the B.Sc. and M.Sc. degrees in elec-

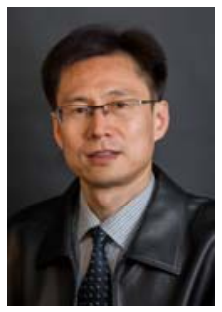
trical engineering from Shandong Polytechnic University, Jinan, China, in 1995 and 1998, respectively, and the $\mathrm{Ph} . \mathrm{D}$. degree in electrical engineering from Shandong University, Jinan, in 2005.

He was a Postdoctoral Researcher with the Department of Computer Science, Shandong University. From 2005 to 2010, he was an Associate Professor with the School of Electrical Engineering, Shandong University, where he has been a Professor since 2010. His research interests include power system analysis and control and smart grids.

Yunhe Hou (M'08-SM'15) received the B.E. and Ph.D. degrees in electrical engineering from Huazhong University of Science and Technology, Wuhan, China, in 1999 and 2005, respectively. He was a Post-Doctoral Research Fellow at Tsinghua University, Beijing, China, from 2005 to 2007, and a Post-Doctoral Researcher at Iowa State University, Ames, IA, USA, and the University College Dublin, Dublin, Ireland, from 2008 to 2009 . He was also a Decision Systems, Massachusetts Institute of Technology, Cambridge, MA, USA, in 2010. He has been a Guest Professor with Huazhong University of Science and Technology, China from 2017 and an Adviser of China Electric Power Research Institute from 2019. He joined the faculty of the University of Hong Kong, Hong Kong, in 2009, where he is currently an Associate Professor with the Department of Electrical and Electronic Engineering. Dr. Hou is an Editor of the IEEE Transactions on Smart Grid and an Associate Editor of the Journal of Modern Power Systems and Clean Energy. 


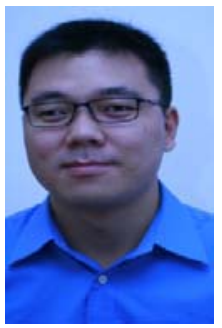

Qiuwei Wu (M'08-SM'15) obtained the PhD degree in Power System Engineering from Nanyang Technological University, Singapore, in 2009.

He was a senior R\&D engineer with VESTAS Technology R\&D Singapore Pte Ltd from Mar. 2008 to Oct. 2009. He has been working at Department of Electrical Engineering, Technical University of Denmark (DTU) since Nov. 2009 (PostDoc Nov. 2009-Oct. 2010, Assistant Professor Nov. 2010-Aug. 2013, Associate Professor since Sept. 2013). He was a visiting scholar at Department of Industrial Engineering \& Operations Research (IEOR), University of California, Berkeley, from Feb. 2012 to May 2012 funded by the Danish Agency for Science, Technology and Innovation (DASTI), Denmark. He was a visiting professor named by Y. Xue, an Academician of Chinese Academy of Engineering, at Shandong University, China, from Nov. 2015 to Oct. 2017. He was a visiting scholar at the Harvard China Project, School of Engineering and Applied Sciences, Harvard University from Nov. 2017 - Oct. 2018 funded by the Otto Mønsted Fond. His research interests are operation and control of power systems with high penetration of renewables, including wind power modelling and control, active distribution networks, and operation of integrated energy systems. He is an Editor of IEEE Transactions on Smart Grid and IEEE Power Engineering Letters. He is also an Associate Editor of International Journal of Electrical Power and Energy Systems, and Journal of Modern Power Systems and Clean Energy. He is a subject editor for IET Generation, Transmission \& Distribution, and IET Renewable Power Generation.

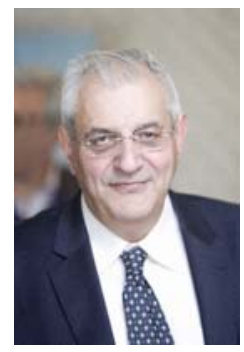

Nikos D. Hatziargyriou is full professor in Power Systems at the Electrical and Computer Engineering School of the National Technical University of Athens. From 2015 to 2019 he was Chair and until 2018, CEO of the Hellenic Distribution Network Operator (HEDNO). From 2007 until 2012 he was executive Vice-Chair and Deputy CEO of the Public Power Corporation (PPC), responsible for the Transmission and Distribution Divisions. He is Fellow Member of IEEE, past Chair of the Power System Dynamic Performance Committee (PSDPC) and currently Editor in Chief of the IEEE Trans on Power Systems. He is honorary member of CIGRE and past Chair of CIGRE SC C6 "Distribution Systems and Distributed Generation". He was chair and currently vice-chair of the EU Technology and Innovation Platform on Smart Networks for Energy Transition (ETIP-SNET). He has participated in more than 60 RD\&D projects funded by the EU Commission, electric utilities and manufacturers. He is author of the book "Microgrids: Architectures and Control" and of more than 250 journal publications and 500 conference proceedings papers. He is included in the 2016, 2017 and 2019 Thomson Reuters lists of the top $1 \%$ most cited researchers.

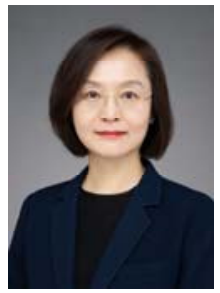

Wen Zhang received the B.S., M.S., and Ph.D. degrees from Shandong University, Jinan, China, in 1989, 1992, and 2006, respectively, all in Electrical Engineering. She is currently a professor with the Key Laboratory of Power System Intelligent Dispatch and Control of Ministry of Education, Shandong University. Her current research interests include power system analysis and control, demand-side response, and application of artificial intelligence to power systems.

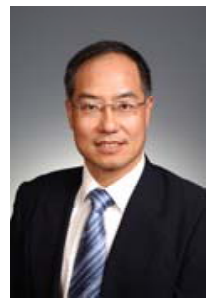

Yutian Liu (SM'96) received the B.E. and M.S. degrees in electrical engineering from the Shandong University of Technology, Jinan, China, in 1984 and 1990, respectively, and the Ph.D. degree in electrical engineering from Xi'an Jiaotong University, Xi'an, China, in 1994.

$\mathrm{He}$ is currently a Chair Professor at the School of Electrical Engineering, Shandong University, Jinan, China. His research interests include power system analysis and control, renewable energy integration, and artificial intelligence application to power system. 